\title{
Differentiability of pseudo-dual-quaternionic functions with a differential operator
}

\author{
Ji Eun Kim
}

Department of Mathematics, Dongguk University, Gyeongju-si 38066, Republic of Korea.

Communicated by R. Saadati

\begin{abstract}
This paper introduces the new concept of pseudo-dual-quaternions and some of their basic properties based on matrices. We extend the concept of differentiability to pseudo-dual-quaternionic functions. Also, we propose a corresponding CauchyRiemann formulas induced the properties of a holomorphic function of pseudo-dual-quaternionic variables.
\end{abstract}

Keywords: Dual-quaternion, differential operators, differentiability, Cauchy-Riemann formulas.

2010 MSC: 32A99, 32W50, 30G35, 11E88.

(C)2018 All rights reserved.

\section{Introduction}

Dual numbers are based on the non-zero and non-real term $\varepsilon$ satisfying $\varepsilon^{2}=0$, instead of $i$ which constitutes complex numbers and satisfies $i^{2}=-1$. Dual numbers are in the two-dimensional form of $x+\varepsilon y$ with base 1 and $\varepsilon$ associative-commutative algebras over the field of real numbers. In 1873, dual numbers introduced by William Clifford [2] are a type of generalized complex numbers. Unlike the field of complex numbers, The algebra of dual numbers comprises zero divisors and is considered over an arbitrary field or commutative ring. By using the structure of dual numbers and the properties of $\varepsilon$, it has been studied in a variety of fields including non-Euclidean geometry. Study [13] defined dual angles having the form of dual numbers to represent the relation between two skew lines in the Euclidean space. Messelmi [10] generalized holomorphicity to dual-complex functions simplifying manipulation of dual-complex numbers, based on matrices. Since then, dual numbers have been applied to study the kinematics, dynamics and open-chain robot manipulators. Dual numbers are useful for geometrical treatments with analytical methods in kinematics and dynamics of spatial mechanisms. Thus, the application of dual numbers in the study of problems in science and engineering is being studied (see $[1,3,12,15])$.

The theory of functions over the complex field has induced the theory for the non-trivial real associative and invertible algebra. In 1843, Hamilton [6] discovered a 4-dimensional division algebra called

Email address: jeunkim@pusan.ac.kr (Ji Eun Kim)

doi: $10.22436 /$ jnsa.011.11.03 
the quaternions with 3 imaginary values which are multiplication provided proper inverses. The quaternionic derivatives of the functions of a quaternion variable can be represented by quaternionic power series similar to power series in four real variables. In 1935, Fueter [4] proposed a definition of regular for quaternionic functions by using the analogue Cauchy-Riemann equations. Also, Fueter [5] led to analogues of Cauchys theorem and Cauchys integral formula. Sudbery [14] gave simple proofs of the main theorems of quaternionic analysis and made clear the relationship between quaternionic analysis and complex analysis by means of the exterior differential calculus. Since then, quaternionic analysis has been investigated by various approaches and calculation processes (see $[7,9,11])$.

In this paper, we combine the unit $\varepsilon$ of dual numbers with the structure of quaternions. From this, we obtain the modified quaternions which is called a pseudo-dual-quaternion (PDQ). Quaternions possess the noncommutative rule of the product and preserve each part of their units after computing operators defined on quaternions. However, PDQs preserve the noncommutative rule of the product and can express the projection of an arbitrary PDQ onto a plane due to the property of the product for PDQs. We have investigated the properties of the unit of dual numbers (see [8]) and we deal with quaternions by means of the unit of dual numbers. This paper introduces PDQs and some of their basic properties. Also, we give an expression of PDQs based on matrices. We extend the concept of holomorphicity and a corresponding Cauchy-Riemann formulas for pseudo-dual-quaternionic functions.

\section{Preliminaries}

The ordinary quaternion is a four-parameter of complex number systems which has two components. Let $\mathbb{D}_{\mathbb{P}}$ denote the set of PDQs, which has elements of the form

$$
p=x_{0}+i x_{1}+j x_{2}+k x_{3}
$$

where $x_{r}(r=0,1,2,3)$ are real, $i$ is the imaginary unit, and $j$ is the unit element such that

$$
i^{2}=-1, \quad j^{2}=0
$$

and let $k:=i j$, then

$$
i j=-j i=k, \quad j k=k j=0, k i=-i k=j,
$$

which is isomorphic to $\mathbb{R}^{4}$. By the properties (2.1) of $i$ and $j$, we have the following rules for an addition and a product that are

$$
p+q=\left(x_{0}+y_{0}\right)+i\left(x_{1}+y_{1}\right)+j\left(x_{2}+y_{2}\right)+k\left(x_{3}+y_{3}\right)
$$

and

$$
p q=\left(x_{0} y_{0}-x_{1} y_{1}\right)+i\left(x_{1} y_{0}+x_{0} y_{1}\right)+j\left(x_{2} y_{0}+x_{3} y_{1}+x_{0} y_{2}-x_{1} y_{3}\right)+k\left(x_{3} y_{0}-x_{2} y_{1}+x_{1} y_{2}+x_{0} y_{3}\right),
$$

respectively. From the definition of the product for PDQs, we give a conjugation of that numbers. The conjugation of each complex number is important to play a role for algebraic and geometric properties of C. For PDQs, we can also extend this notion. Let $p=x_{0}+i x_{1}+j x_{2}+k x_{3}$ be a PDQ. Then we define the conjugate of $p$ by the formula

$$
p^{*}=x_{0}-i x_{1}-j x_{2}-k x_{3}
$$

where $p$ has the following relation

$$
p p^{*}=p^{*} p \in \mathbb{R} \text {. }
$$

Thus, we have the modulus, denoted by $\mathcal{N}(p)$, and the inverse element, denoted by $\frac{1}{p}$ of $p \in \mathcal{D}_{p}$ :

$$
\mathcal{N}(p):=p p^{*}=x_{0}^{2}+x_{1}^{2}
$$


and

$$
\frac{1}{p}=\frac{p^{*}}{\mathcal{N}(p)}=\frac{x_{0}}{x_{0}^{2}+x_{1}^{2}}-i \frac{x_{1}}{x_{0}^{2}+x_{1}^{2}}-j \frac{x_{2}}{x_{0}^{2}+x_{1}^{2}}-k \frac{x_{3}}{x_{0}^{2}+x_{1}^{2}}, \quad\left(x_{0}, x_{1} \neq 0\right),
$$

respectively. Furthermore, $\sqrt{\mathcal{N}(p)}$ is expressed by Figure 1 .

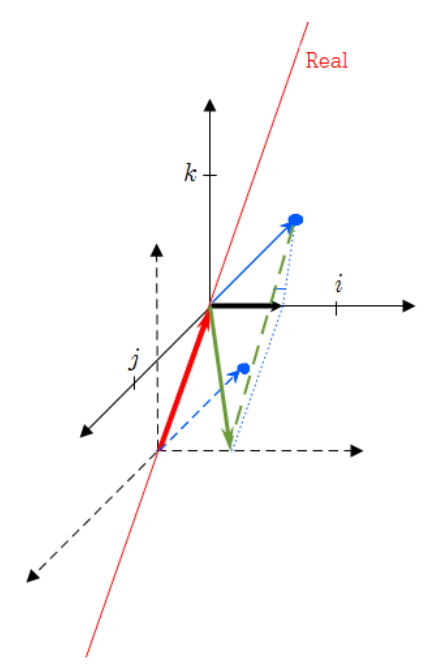

Figure 1: $\sqrt{\mathcal{N}(p)}$ is the green vector which is the orthographic projection of the blue line onto the plane consisting of the red line and the line containing $i$.

PDQs form a non-commutative ring give the structure of the four dimensional real Algebra. The algebra $\mathcal{D}_{\mathrm{p}}$ is not a division algebra since if it has the elements satisfying $x_{0}^{2}+x_{1}^{2}=0$, that is, if $x_{0}=x_{1}=0$, the element is not invertible. All elements with the above property are zero divisors. Then, we introduce the set $\mathcal{D}_{0}$ of zero divisors of $\mathcal{D}_{\mathfrak{p}}$ which can be called the null part of $\mathcal{D}_{\mathfrak{p}}$ as

$$
\mathcal{D}_{0}=\left\{p \in \mathcal{D}_{p} \mid p=x_{0}+i x_{1}+j x_{2}+k x_{3}, \quad x_{0}^{2}+x_{1}^{2}=0\right\}=\left\{p \in \mathcal{D}_{p} \mid p=j x_{2}+k x_{3}, \quad x_{2}, x_{3} \in \mathbb{R}\right\} .
$$

There can be also the representation such as

$$
i=\left(\begin{array}{cc}
i & 0 \\
0 & -i
\end{array}\right), \quad j=\left(\begin{array}{ll}
0 & 1 \\
0 & 0
\end{array}\right), \quad k=\left(\begin{array}{ll}
0 & i \\
0 & 0
\end{array}\right) .
$$

So, PDQs can be also written by using matrices with the above units:

$$
p=\left(\begin{array}{cc}
z_{1} & z_{2} \\
0 & \overline{z_{1}}
\end{array}\right)
$$

where $z_{1}=x_{0}+i x_{1}, z_{2}=x_{2}+i x_{3}$ and $\overline{z_{1}}=x_{0}-i x_{1}$.

We let the set of PDQs expressed by matrices, which is denoted by $\mathbb{M}_{\delta}$,

$$
\mathbb{M}_{\delta}=\left\{A \in M_{2}(\mathbb{C}) \mid A=\left(\begin{array}{cc}
z_{1} & z_{2} \\
0 & \overline{z_{1}}
\end{array}\right)\right\}
$$

Thus, the set $\mathbb{M}_{\delta}$ is a subring of $M_{2}(\mathbb{C})$ which forms a four dimensional real associative and noncommutative Algebra. Let us now define the map

$$
\mathcal{T}_{\delta}: \mathcal{D}_{\mathrm{p}} \rightarrow \mathbb{M}_{\delta}, \quad \mathcal{T}_{\delta}(p)=\left(\begin{array}{cc}
z_{1} & z_{2} \\
0 & \overline{z_{1}}
\end{array}\right)
$$

From the definition of the map $\mathcal{T}_{\delta}$, we can give a correspondence between the two algebras $\mathcal{D}_{\mathrm{p}}$ and $\mathbb{M}_{\delta}$ via the map $\mathcal{T}_{\delta}$. Furthermore, the map $\mathcal{T}_{\delta}$ is an isomorphism of algebras. 
Proposition 2.1. We also note that $\mathrm{p} \in \mathcal{D}_{0}$ if and only if $\operatorname{det}\left(\mathcal{T}_{\delta}(\mathrm{p})\right)=0$.

Proof. Suppose that $p \in \mathcal{D}_{0}$. Then $p=j x_{2}+k x_{3}, x_{2}, x_{3} \in \mathbb{R}$, that is, for $p=x_{0}+i x_{1}+j x_{2}+k x_{3}, x_{0}=x_{1}=0$. Hence, we have

$$
\operatorname{det}\left(\mathcal{T}_{\delta}(p)\right)=\left|\begin{array}{cc}
z_{1} & z_{2} \\
0 & \overline{z_{1}}
\end{array}\right|=x_{0}^{2}+x_{1}^{2}=0
$$

Conversely, by the definition of $\mathcal{D}_{0}$ and $\mathcal{T}_{\delta}$, if $\operatorname{det}\left(\mathcal{T}_{\delta}(p)\right)=0$, then $x_{0}=x_{1}=0$. Thus, $p$ has the form $p=j x_{2}+k x_{3} \in \mathcal{D}_{0}$.

Now, we give the map, denoted by $|.|_{\delta}$ :

$$
\text { |.|⿰ }:_{\delta} \mathcal{D}_{\mathrm{p}} \rightarrow \mathbb{R}^{+}, \quad|p|_{\delta}=\left|z_{1} \overline{z_{1}}\right|^{1 / 2}=\sqrt{x_{0}^{2}+x_{1}^{2}},
$$

where $\mathbb{R}^{+}$is the set of positive real numbers.

Proposition 2.2. The map $\left.\left.\right|_{\mid}\right|_{\delta}$ has the following properties:

(i) $|p|_{\delta}=\left|\operatorname{det}\left(\mathcal{T}_{\delta}(p)\right)\right|^{1 / 2}$ for $p \in \mathcal{D}_{p}$;

(ii) $|\mathrm{p}+\mathrm{q}|_{\delta} \leqslant|\mathrm{p}|_{\delta}+|\mathrm{q}|_{\delta}$ for $\mathrm{p}, \mathrm{q} \in \mathcal{D}_{\mathrm{p}}$;

(iii) $|\mathrm{pq}|_{\delta}=|\mathrm{p}|_{\delta}|\mathrm{q}|_{\delta}$ for $\mathrm{p}, \mathrm{q} \in \mathcal{D}_{\mathrm{p}}$;

(iv) $|\alpha p|_{\delta}=|\alpha||p|_{\delta}$ for $p \in \mathcal{D}_{p}$;

(v) $|p|_{\delta}=0$ if and only if $p \in \mathcal{D}_{0}$,

where |.| is the usual norm in complex analysis.

The proofs of the above properties are an immediate consequence of the definition of $|.|_{\delta}$ and their calculations. Thus, we can induce a structure of a corresponding topology over the algebra $\mathcal{D}_{\mathrm{p}}$. So, we construct a pseudo-dual disk and pseudo-dual sphere of center $p_{0}=x_{0}^{o}+i x_{1}^{o}+j x_{2}^{o}+k x_{3}^{o}$ and radius $r>0$, in $\mathcal{D}_{p}$, by

$$
D_{\delta}\left(p_{0}, r\right):=\left\{p=x_{0}+i x_{1}+j x_{2}+k x_{3} \in \mathcal{D}_{p}|| p-\left.p_{0}\right|_{\delta}<r\right\}
$$

and

$$
S_{\delta}\left(p_{0}, r\right):=\left\{p=x_{0}+i x_{1}+j x_{2}+k x_{3} \in \mathcal{D}_{p}|| p-\left.p_{0}\right|_{\delta}=r\right\}
$$

respectively.

\section{Hyperholomorphicity of pseudo-dual-quaternionic functions}

We consider some properties of functions of pseudo-dual-quaternionic variable. We investigate the continuity of pseudo-dual-quaternionic functions and the holomorphicity in those functions.

Definition 3.1. Let $\Omega$ be an open subset of $\mathcal{D}_{p}$. Then for $p=x_{0}+i x_{1}+j x_{2}+k x_{3}$, a function $f: \Omega \rightarrow \mathcal{D}_{p}$,

$$
f(p)=u_{0}+i u_{1}+j u_{2}+k u_{3}
$$

where $u_{t}=u_{t}\left(x_{0}, x_{1}, x_{2}, x_{3}\right)(t=0,1,2,3)$ are real-valued functions, is said to be a pseudo-dual-quaternionic function (PDQ-function) if it is a mapping from a subset $\Omega$ of $\mathcal{D}_{\mathrm{p}}$ to $\mathcal{D}_{\mathrm{p}}$.

Definition 3.2. Let $\Omega$ be an open subset of $\mathcal{D}_{\mathrm{p}}$. Then for $p \in \Omega$, a PDQ-function $f: \Omega \rightarrow \mathcal{D}_{\mathrm{p}}$ is said to be continuous at $p_{0}=x_{0}^{o}+i x_{1}^{o}+j x_{2}^{o}+k x_{3}^{o}$ if there exists the limit satisfying

$$
\lim _{p \rightarrow p_{0}} f(p)=f\left(p_{0}\right),
$$

where the limit is calculated for each component, 


$$
\lim _{p \rightarrow p_{0}} f(p)=\lim _{\substack{x_{t} \rightarrow x_{t}^{o} \\(t=0,1,2,3)}} f(p)=f\left(p_{0}\right)
$$

That is, for given $\varepsilon>0$ there exists $\delta>0$ such that each $\left|x_{t}-x_{t}^{o}\right|<\delta(t=0,1,2,3)$ implies $\left|u_{r}-u_{r}^{o}\right|<\varepsilon$ $(r=0,1,2,3)$, where $u_{r}^{o}=u_{r}\left(x_{0}^{o}, x_{1}^{o}, x_{2}^{o}, x_{3}^{o}\right)$. Moreover, the function $f$ is continuous in $\Omega$ if $f$ is continuous at every point of $\Omega$.

Definition 3.3. Let $\Omega$ be an open subset of $\mathcal{D}_{\mathrm{p}}$. Then for $p \in \Omega$, a PDQ-function $f: \Omega \rightarrow \mathcal{D}_{\mathrm{p}}$ is said to be left-differentiable at $p_{0}=x_{0}^{o}+i x_{1}^{o}+j x_{2}^{o}+k x_{3}^{o}$ if the limit exists

$$
\frac{d f}{d p}\left(p_{0}\right)_{L}:=\lim _{p \rightarrow p_{0}} \frac{1}{p-p_{0}}\left\{f(p)-f\left(p_{0}\right)\right\},
$$

where the limit is also calculated for each component, and $f$ is said to be left-differentiable at $p_{0}$ if the limit exists

$$
\frac{d f}{d p}\left(p_{0}\right)_{R}:=\lim _{p \rightarrow p_{0}}\left\{f(p)-f\left(p_{0}\right)\right\} \frac{1}{p-p_{0}},
$$

where the limit is also calculated for each component. Here $\frac{d f}{d p}\left(p_{0}\right)_{L}$ is called the left-derivative and $\frac{d f}{d p}\left(p_{0}\right)_{R}$ is called the right-derivative of $f$ at the point $p_{0}$, respectively. Moreover, if $f$ is left(right)differentiable for every point in a neighborhood of the point $p_{0}$, then $f$ is called left(right)-holomorphic at $p_{0}$. The function $f$ is left(right)-holomorphic in $\Omega$ if $f$ is left(right)-holomorphic at every point of $\Omega$.

Since PDQs have the non-commutative rule of product, we need to consider the order of multiplication. So we give two definitions of differentiable functions such as the left-differentiability and the right-differentiability. The right differentiability is similar to the left differentiability with respect to the property and the process of calculations, so we deal with the left-differentiability. In addition, the derivative that can be expressed later is the left-differentiable, and for convenience it is expressed as

$$
\frac{d f}{d p}\left(p_{0}\right)_{L}:=\frac{d f}{d p}\left(p_{0}\right) \text { and } \lim _{p \rightarrow p_{0}} \frac{1}{p-p_{0}}\left\{f(p)-f\left(p_{0}\right)\right\}:=\lim _{p \rightarrow p_{0}} \frac{f(p)-f\left(p_{0}\right)}{p-p_{0}} .
$$

Now, we generalize a corresponding Cauchy-Riemann formulas to PDQ-functions.

Theorem 3.4. Let $\Omega$ be an open subset of $\mathcal{D}_{\mathrm{p}}$ and $\mathrm{f}$ be a PDQ-function in $\Omega$. If the function $\mathrm{f}$ is holomorphic at $p_{0}$, then $\mathrm{f}$ satisfies

$$
\frac{d f}{d p} i=\frac{\partial f}{\partial x_{1}}, \quad \frac{d f}{d p} j=\frac{\partial f}{\partial x_{2}}, \quad \frac{d f}{d p} k=\frac{\partial f}{\partial x_{3}}, \quad \text { and } \quad \frac{d f}{d p}=\frac{\partial f}{\partial x_{0}} .
$$

Proof. Suppose $f$ is holomorphic at $p_{0}$. Then there exists the limit $\frac{d f}{d p}\left(p_{0}\right)$. So, we can express as follows:

$$
\begin{aligned}
\frac{\partial f}{\partial x_{0}}= & \lim _{\substack{x_{\mathfrak{t}} \rightarrow x_{0}^{o} \\
(t=0 ; 1 ; 2 ; 3)}} \frac{f\left(x_{0}+i x_{1}^{o}+j x_{2}^{o}+k x_{3}^{o}\right)-f\left(x_{0}^{o}+i x_{1}^{o}+j x_{2}^{o}+k x_{3}^{o}\right)}{x_{0}-x_{0}^{o}} \\
= & \lim _{\substack{x_{\mathfrak{t}} \rightarrow x_{0}^{o} \\
(t=0 ; 1 ; 2 ; 3)}} \frac{f\left(x_{0}+i x_{1}+j x_{2}+k x_{3}\right)-f\left(x_{0}^{o}+i x_{1}^{o}+j x_{2}^{o}+k x_{3}^{o}\right)}{x_{0}-x_{0}^{o}} \\
& +\lim _{\substack{x_{\mathfrak{t}} \rightarrow x_{0}^{o} \\
(t=0 ; 1 ; 2 ; 3)}} \frac{f\left(x_{0}+i x_{1}^{o}+j x_{2}^{o}+k x_{3}^{o}\right)-f\left(x_{0}+i x_{1}+j x_{2}^{o}+k x_{3}^{o}\right)}{x_{0}-x_{0}^{o}} \\
& +\lim _{\substack{x_{\mathfrak{t}} \rightarrow x_{0}^{o} \\
(t=0 ; 1 ; ; ; 3)}} \frac{f\left(x_{0}+i x_{1}+j x_{2}^{o}+k x_{3}^{o}\right)-f\left(x_{0}+i x_{1}+j x_{2}+k x_{3}^{o}\right)}{x_{0}-x_{0}^{o}} \\
& +\lim _{\substack{x_{t} \rightarrow x_{0}^{o} \\
(t=0 ; 1 ; 2 ; 3)}} \frac{f\left(x_{0}+i x_{1}+j x_{2}+k x_{3}^{o}\right)-f\left(x_{0}+i x_{1}+j x_{2}+k x_{3}\right)}{x_{0}-x_{0}^{o}}
\end{aligned}
$$




$$
\begin{aligned}
= & \lim _{p \rightarrow p_{0}} \frac{f(p)-f\left(p_{0}\right)}{p_{0}-p_{0}} \frac{p_{0}-p_{0}}{x_{0}-x_{0}^{o}}-\lim _{\substack{x_{t} \rightarrow x_{0}^{o} \\
(t=0 ; 1 ; 2 ; 3)}} \frac{f\left(x_{0}+i x_{1}+j x_{2}^{o}+k x_{3}^{o}\right)-f\left(x_{0}+i x_{1}^{o}+j x_{2}^{o}+k x_{3}^{o}\right)}{x_{1}-x_{1}^{o}} \frac{x_{1}-x_{1}^{o}}{x_{0}-x_{0}^{o}} \\
& -\lim _{\substack{x_{t} \rightarrow x_{0}^{o} \\
(t=0 ; 1 ; 2 ; 3)}} \frac{f\left(x_{0}+i x_{1}+j x_{2}+k x_{3}^{o}\right)-f\left(x_{0}+i x_{1}+j x_{2}^{o}+k x_{3}^{o}\right)}{x_{2}-x_{2}^{o}} \frac{x_{2}-x_{2}^{o}}{x_{0}-x_{0}^{o}} \\
& -\lim _{\substack{x_{t} \rightarrow x_{0}^{o} \\
(t=0 ; 1 ; 2 ; 3)}} \frac{f\left(x_{0}+i x_{1}+j x_{2}+k x_{3}\right)-f\left(x_{0}+i x_{1}+j x_{2}+k x_{3}^{o}\right)}{x_{3}-x_{3}^{o}} \frac{x_{3}-x_{3}^{o}}{x_{0}-x_{0}^{o}} .
\end{aligned}
$$

Since we have

$$
\lim _{p \rightarrow p_{0}} \frac{f(p)-f\left(p_{0}\right)}{p_{0}-p_{0}} \frac{p_{0}-p_{0}}{x_{0}-x_{0}^{o}}=\lim _{p \rightarrow p_{0}} \frac{f(p)-f\left(p_{0}\right)}{p_{0}-p_{0}} \frac{\left(x_{0}-x_{0}^{o}\right)+\left(x_{1}-x_{1}^{o}\right) i+\left(x_{2}-x_{2}^{o}\right) j+\left(x_{3}-x_{3}^{o}\right) k}{x_{0}-x_{0}^{o}},
$$

we obtain

$$
\frac{\partial f}{\partial x_{0}}=\frac{d f}{d p}+\left(\frac{d f}{d p} i-\frac{\partial f}{\partial x_{1}}\right) \frac{x_{1}-x_{1}^{o}}{x_{0}-x_{0}^{o}}+\left(\frac{d f}{d p} j-\frac{\partial f}{\partial x_{2}}\right) \frac{x_{2}-x_{2}^{o}}{x_{0}-x_{0}^{o}}+\left(\frac{d f}{d p} k-\frac{\partial f}{\partial x_{3}}\right) \frac{x_{3}-x_{3}^{o}}{x_{0}-x_{0}^{o}} .
$$

Since $f$ is holomorphic at $p_{0}$, in order to determine the existence of the limit $\frac{d f}{d p}$, the limits

$$
\left(\frac{d f}{d p} i-\frac{\partial f}{\partial x_{1}}\right), \quad\left(\frac{d f}{d p} j-\frac{\partial f}{\partial x_{2}}\right), \quad \text { and }\left(\frac{d f}{d p} k-\frac{\partial f}{\partial x_{3}}\right)
$$

should be independent to ratios

$$
\frac{x_{1}-x_{1}^{\mathrm{o}}}{x_{0}-x_{0}^{\mathrm{o}}}, \frac{x_{2}-x_{2}^{\mathrm{o}}}{x_{0}-x_{0}^{\mathrm{o}}}, \text { and } \frac{x_{3}-x_{3}^{\mathrm{o}}}{x_{0}-x_{0}^{\mathrm{o}}}
$$

respectively, and then

$$
\frac{\partial f}{\partial x_{0}}=\frac{d f}{d p}
$$

Thus, the equations

$$
\frac{d f}{d p} i=\frac{\partial f}{\partial x_{1}}, \quad \frac{d f}{d p} j=\frac{\partial f}{\partial x_{2}}, \quad \frac{d f}{d p} k=\frac{\partial f}{\partial x_{3}}, \quad \text { and } \quad \frac{\partial f}{\partial x_{0}}=\frac{d f}{d p}
$$

are obtained.

Theorem 3.5. Let $\Omega$ be an open subset of $\mathcal{D}_{\mathrm{p}}$ and $\mathrm{f}$ be a PDQ-function in $\Omega$. A function $\mathrm{f}$ is holomorphic on $\Omega$ if and only if the function $\mathrm{f}$ satisfies

$$
D^{*} f=0,
$$

where the differential operator $\mathrm{D}^{*}$ is

$$
\mathrm{D}^{*}=\vec{v} \frac{\partial}{\partial x_{0}}-\frac{\partial}{\partial x_{1}}-\frac{\partial}{\partial x_{2}}-\frac{\partial}{\partial x_{3}}
$$

with $\vec{v}=i+j+k$ satisfying $\vec{v}^{2}=-1$.

Proof. Since the function $f$ is holomorphic on $\Omega$, the following equations is derived from Theorem 3.4.

$$
\frac{\partial f}{\partial x_{0}} i=\frac{\partial f}{\partial x_{1}}, \quad \frac{\partial f}{\partial x_{0}} j=\frac{\partial f}{\partial x_{2}}, \quad \frac{\partial f}{\partial x_{0}} k=\frac{\partial f}{\partial x_{3}} .
$$

If the above equations are detailed, then we have

$$
\frac{\partial u_{0}}{\partial x_{0}}=\frac{\partial u_{r}}{\partial x_{r}} \quad(r=1,2,3),
$$




$$
\begin{aligned}
\frac{\partial u_{r}}{\partial x_{t}}=0 \quad(r=0,1 ; t=2,3), \\
\frac{\partial u_{0}}{\partial x_{1}}+\frac{\partial u_{1}}{\partial x_{0}}=0, \quad \frac{\partial u_{2}}{\partial x_{0}}+\frac{\partial u_{3}}{\partial x_{1}}=0, \quad \frac{\partial u_{1}}{\partial x_{0}}+\frac{\partial u_{2}}{\partial x_{3}}=0 \\
\frac{\partial u_{3}}{\partial x_{2}}-\frac{\partial u_{1}}{\partial x_{0}}=0, \quad \frac{\partial u_{3}}{\partial x_{0}}-\frac{\partial u_{2}}{\partial x_{1}}=0 .
\end{aligned}
$$

That is, we have

$$
\left\{\begin{array}{l}
\frac{\partial u_{0}}{\partial x_{0}}=\frac{\partial u_{r}}{\partial x_{r}}, \quad(r=1,2,3) \\
\frac{\partial u_{r}}{\partial x_{t}}=0, \quad(r=0,1 ; t=2,3), \\
\frac{\partial u_{0}}{\partial x_{1}}=\frac{\partial u_{2}}{\partial x_{3}}=-\frac{\partial u_{3}}{\partial x_{2}}=-\frac{\partial u_{1}}{\partial x_{0}} \\
\frac{\partial u_{2}}{\partial x_{0}}=-\frac{\partial u_{3}}{\partial x_{1}}, \\
\frac{\partial u_{3}}{\partial x_{0}}=\frac{\partial u_{2}}{\partial x_{1}}
\end{array}\right.
$$

Comparing the result of the calculation of

$$
D^{*} f=\vec{v} \frac{\partial f}{\partial x_{0}}-\frac{\partial f}{\partial x_{1}}-\frac{\partial f}{\partial x_{2}}-\frac{\partial f}{\partial x_{3}}
$$

with the equations (3.1), we can obtain that the holomorphic function $f$ satisfies $D^{*} f=0$.

Corollary 3.6. Let $\Omega$ be an open subset of $\mathcal{D}_{\mathrm{p}}$. Suppose that $\mathrm{f}$ is holomorphic and continuously differentiable in a domain $\Omega$ with differentiable boundary. Then the function $\mathrm{f}$ satisfies

$$
\int_{\partial \Omega} D^{*} f=0
$$

Proof. Cauchys theorem holds for any contour of integration. The integral theorems, for holomorphic PDQ-functions with the values of the interior of a region in terms of its values on the boundary, holds for a general rectifiable boundary. From this, applying the Stokes theorem, it follows that if $f$ is holomorphic and continuously differentiable in a domain $\Omega$ with differentiable boundary, then the function $f$ satisfies $D^{*} f=0$ and thus, we obtain

$$
\int_{\partial \Omega} D^{*} f=0
$$

\section{Conclusion}

In this paper, the algebraic and analytic properties of the modified quaternions, called a pseudo-dualquaternion, is obtained. Like the rule of the product for quaternions, and PDQs preserve the noncommutative rule of the product. However, since PDQs can express the projection of an arbitrary PDQ onto a plane due to the property of the product for PDQs, the function of PDQ variables can be extended to the new concept of holomorphicity of PDQ functions. Thus, we have defined derivatives to be applied to the PDQ system. A calculation method using a formula called a corresponding Cauchy-Riemann formulas can be also substituted for the definition of derivative. And, the theorem related to this definition of derivative can be proved using the corresponding Cauchy-Riemann formulas. Therefore, the mathematical approach based on the function over the PDQ system and its application to the rate of change such as the velocity of objects and the slope of the tangent line can be induced.

\section{Acknowledgment}

This work was supported by the Dongguk University Research Fund of 2017. 


\section{References}

[1] H. H. Cheng, Programming with dual numbers and its applications in mechanisms design, Eng. Comput., 10 (1994), 212-229. 1

[2] M. A. Clifford, Preliminary Sketch of Biquaternions, Proc. Lond. Math. Soc., 4 (1871/73), 381-395. 1

[3] J. R. Dooley, J. M. McCarthy, Spatial rigid body dynamics using dual quaternion components, Proceedings. 1991 IEEE International Conference on Robotics and Automation, 1991 (1991), 90-95. 1

[4] R. Fueter, Die Funktionentheorie der Differentialgleichungen $\Delta \mathrm{u}=0$ und $\Delta \Delta \mathrm{u}=0$ mit vier reellen Variablen, Comment. math. Helv., 7 (1934), 307-330. 1

[5] R. Fueter, Über die analytische Darstellung der regulären Funktionen einer Quaternionenvariablen, Comment. math. Helv., 8 (1935), 371-378. 1

[6] W. R. Hamilton, Elements of Quaternions, Longmans, Green, and Company, London, (1899). 1

[7] J. J. Kajiwara, X. D. Li, K. H. Shon, Regeneration in Complex, Quaternion and Clifford analysis, in: Finite or Infinite Dimensional Complex Analysis and Applications, 2004 (2004), 287-298. 1

[8] J. E. Kim, The corresponding inverse of functions of multidual complex variables in Clifford analysis, J. Nonlinear Sci. Appl., 9 (2016), 4520-4528. 1

[9] J. E. Kim, A corresponding Cullen-regularity for split-quaternionic-valued functions, Adv. Difference Equ., 2017 (2017), 14 pages. 1

[10] F. Messelmi, Dual-complex numbers and their holomorphic functions, HAL archives-ouvertes.fr, Centre Pour la Comm. Scientifique Directe, 2015 (2015), 11 pages. 1

[11] K. Nôno, Hyperholomorphic functions of a quaternion variable, Bull. Fukuoka Univ. Ed. III, 32 (1982), 21-37. 1

[12] B. Ravani, B. Roth, Mappings of spatial kinematics, J. Mech., Trans., and Automation, 106 (1984), 341-347. 1

[13] E. Study, Geometrie der Dynamen, BG Teubner, Leipzig, (1903). 1

[14] A. Sudbery, Quaternionic analysis, Math. Proc. Cambridge Philos. Soc., 85 (1979), 199-225. 1

[15] A. T. Yang, F. Freudenstein, Application of dual-number quaternion algebra to the analysis of spatial mechanisms, J. Appl. Mech., 31 (1964), 300-308. 1 